\title{
Dynamics of a Virological Model for Cancer Therapy with Innate Immune Response
}

\author{
Ayoub Nouni, ${ }^{1}$ Khalid Hattaf $\mathbb{D}^{1,2}$ and Noura Yousfi ${ }^{1}$ \\ ${ }^{1}$ Laboratory of Analysis, Modeling and Simulation (LAMS), Faculty of Sciences Ben M'sik, Hassan II University of Casablanca, \\ P.O. Box 7955 Sidi Othman, Casablanca, Morocco \\ ${ }^{2}$ Centre Régional des Métiers de l'Education et de la Formation (CRMEF), 20340 Derb Ghalef, Casablanca, Morocco
}

Correspondence should be addressed to Khalid Hattaf; k.hattaf@yahoo.fr

Received 22 June 2020; Revised 24 August 2020; Accepted 12 September 2020; Published 27 September 2020

Academic Editor: Frédéric Magoulès

Copyright (c) 2020 Ayoub Nouni et al. This is an open access article distributed under the Creative Commons Attribution License, which permits unrestricted use, distribution, and reproduction in any medium, provided the original work is properly cited.

\begin{abstract}
The aim of this work is to present a virological model for cancer therapy that includes the innate immune response and saturation effect. The presented model combines both the evolution of a logistic growing tumor and time delay which stands for the period of the viral lytic cycle. We use the delay differential equation in order to model this time which also means the time needed for the infected tumor cells to produce new virions after viral entry. We show that the delayed model has four equilibria which are the desired outcome therapy equilibrium, the complete failure therapy equilibrium, the partial success therapy free-immune equilibrium when the innate immune response has not been established, and the partial success therapy equilibrium with immune response. Furthermore, the stability analysis of equilibria and the Hopf bifurcation are properly exhibited.
\end{abstract}

\section{Introduction}

Combination therapy approaches have shown a serious promise to deal with cancers that are resistant to traditional therapeutic procedures. Oncolytic virotherapy, also called the selective therapy, is a developable technique that adopts replication competent viruses as a new treatment to destroy cancer cells without causing damage to normal cells [1-4]. Different researches have investigated combination strategies with oncolytic virotherapy and chemotherapeutic drugs in order to optimize both the effect of the added therapy and the viral oncolysis $[5,6]$. Many mathematical models of viral infection and immune response have been established in order to study the behavior as well as the dynamics of the cancer cells. According to Phan and Tian [7], the dynamics of the system, when they considered a composed ODE's dimensional model, is ruled by the viral burst size and some parameters in relation to the innate immune response. They have shown that getting the immune response involved in the system makes the oncolytic virotherapy more difficult by establishing more equilibria when the viral burst size is lower than a critical value, whereas the model has the same behavior like in the case when the immune response is excluded when the viral burst size is big. In 2018, Kim et al. [8] proposed a delayed mathematical model with two controls to describe cancer viral therapy dynamics and to reduce total tumor cell numbers as well as the costs of two therapies. They performed the stability analysis and the existence of Hopf bifurcation. They also inspected the optimal oncolytic immunotherapy treatment with respect to the time delay. However, it is important to notice that either these works did not incorporate the nonlinear relationship between viral dose and infection rate or they did not consider the survival probability of the infected cells during the latent period not to mention the absorbtion rate of the virus. Recently, Hattaf [9] proposed a virological model that incorporates the general infection rate for the two types of transmission, humoral immunity, and three time delays. He found that the entire behavior of the presented model is determined and ruled by the basic reproduction number and the reproduction number for humoral immunity. The study investigated the dynamical behaviors of the model including Hopf bifurcation and stability switches. 
Based on the above and the model for oncolytic virotherapy [10], we propose the following model:

$$
\left\{\begin{array}{l}
\frac{\mathrm{d} x(t)}{\mathrm{d} t}=r x(t)\left(1-\frac{x(t)+y(t)}{K}\right)-\left(\frac{\beta x(t) v(t)}{1+\alpha v(t)}\right), \\
\frac{\mathrm{d} y(t)}{\mathrm{d} t}=\frac{\beta x(t-\tau) v(t-\tau) e^{-m \tau}}{1+\alpha v(t-\tau)}-\delta y(t)-p y(t) z(t), \\
\frac{\mathrm{d} v(t)}{\mathrm{d} t}=N \delta y(t)-\frac{n \beta x(t) v(t)}{1+\alpha v(t)}-\mu v(t)-q v(t) z(t), \\
\frac{\mathrm{d} z(t)}{\mathrm{d} t}=c y(t) z(z)-b z(t),
\end{array}\right.
$$

where $x(t), y(t)$, and $v(t)$ have the same meaning as in [10] which are, respectively, the quantities of uninfected tumor cells, damaged tumor cells, and oncolytic virus. On the other hand, $z(t)$ denotes the concentration of the innate immune cells at time $t$. The tumor grows logistically at a rate $r$ and $K$ is the maximal carrying capacity of tumor cells. The infection rate in the oncoviral therapy model [7] has been modeled by a bilinear incidence which is not reasonable in case of a high concentration of oncolytic virus. Therefore, it is very reasonable to model the infection rate by a saturated incidence of the form $(\beta x v) /(1+\alpha v)$, where $\beta$ and $\alpha$ are positive constants which, respectively, describe the infection process and the saturation effect. The parameters $\delta$ and $\mu$ represent, respectively, the death rate of the damaged tumor cells and the virus clearance rate while $n$ describes the absorbtion rate of the virus in the extracellular tissue, whereas $N$ is the number of new viruses which appear after a disruption-lysis of a damaged tumor cell. The constants $p$ and $q$ stand for the immune killing rate of damaged cells and viruses, respectively, while $b$ represents the immune clearance rate and $c$ is the incitement rate of the innate immune system. Ultimately, the delay $\tau$ expresses the time of latent period while the quantity $e^{-m \tau}$ exemplifies the probability of getting through from time $t-\tau$ to time $t$, where $m$ is the death rate for unproductive-damaged cells.

Our purpose in this work is to extend our model in [10] by introducing the role of the innate immune response in oncolytic virotherapy. Additionally, the model studied in [7] is a special case of system (1) when the time period of the lytic cycle and saturation effect are not considered, that is, $\tau=0$ and $\alpha=0$. Otherwise, this paper is organized as follows. In Section 2, we give some preliminary results and we discuss the conditions of the existence of equilibria. Section 3 deals with stability analysis and provides conditions under which the system undergoes the Hopf bifurcation. Finally, Section 4 is devoted to discussion and conclusion.

\section{Positiveness, Boundedness, and Equilibria}

In this section, we prove the positivity and the boundedness of solutions of model (1).

Let $\mathscr{C}=C\left([-\tau, 0], \mathrm{IR}^{4}\right)$ be the Banach space of continuous functions mapping the interval $[-\tau, 0]$ into $\operatorname{IR}^{4}$ with the topology of the uniform convergence. According to the fundamental theory of functional differential equations [11], model (1) has a unique solution $(x(t), y(t), v(t), z(t))$ with respect to initial values $\left(x_{0}, y_{0}, v_{0}, z_{0}\right) \in \mathscr{C}$. Additionally, we also assume that the initial values satisfy the following biological conditions:

$$
\begin{aligned}
x_{0}(\theta) & \geq 0, \\
y_{0}(\theta) & \geq 0, \\
v_{0}(\theta) & \geq 0, \\
z_{0}(\theta) & \geq 0, \\
\theta & \in[-\tau, 0] .
\end{aligned}
$$

Theorem 1. Each solution of model (1) starting from initial condition (2) remains positive and bounded for all $t \geq 0$.

Proof. From the first and the fourth equations of (1), we get

$$
\begin{aligned}
& x(t)=x(0) e^{\int_{0}^{t} r(1-x(s)+y(s) / K)-\beta v(s) / 1+\alpha v(s) \mathrm{d} s}, \\
& z(t)=z(0) e^{-b t+\int_{0}^{t} c y(s) \mathrm{d} s},
\end{aligned}
$$

which leads to $x(t) \geq 0$ and $z(t) \geq 0$ for all $t \geq 0$. From the second and third equations of (1), we have

$$
\begin{aligned}
& y(t)=y(0) e^{-\delta t-\int_{0}^{t} p z(s) \mathrm{d} s}+e^{-m \tau-\delta t-\int_{0}^{t} p z(s) \mathrm{d} s} \int_{0}^{t} \frac{\beta x(s-\tau) v(s-\tau)}{1+\alpha v(s-\tau)} e^{\delta s+\int_{0}^{s} p z(u) \mathrm{d} u} \mathrm{~d} s, \\
& v(t)=\left(v(0) e^{-\int_{0}^{t} n \beta x(s) / 1+\alpha v(s)+q z(s) \mathrm{d} s}+N \delta \int_{0}^{t} y(u) e^{\mu u-\int_{u}^{t} n \beta x(s) / 1+\alpha v(s)+q z(s) \mathrm{d} s} \mathrm{~d} u\right) e^{-\mu t} .
\end{aligned}
$$


Clearly, $y(t) \geq 0$ and $v(t) \geq 0$ for $t \in[0, \tau]$. This procedure can be repeated on the interval $[\eta \tau,(\eta+1) \tau]$ for all $\eta \in \mathrm{IN}$. Then, $y(t) \geq 0$ and $v(t) \geq 0$ for all $t \geq 0$.

Through the first equation of (1), we have

$$
\frac{\mathrm{d} x(t)}{\mathrm{d} t} \leq r x(t)\left(1-\frac{x(t)}{K}\right)
$$

Applying the comparison principle, we get

$$
\limsup _{t \rightarrow+\infty} x(t) \leq K .
$$

Therefore, $x(t)$ is bounded. Let

$$
T(t)=x(t-\tau) e^{-m \tau}+y(t)+\frac{p}{c} z(t) .
$$

For $t>\tau$, we have

$$
\begin{aligned}
\frac{\mathrm{d} T(t)}{\mathrm{d} t}= & r x(t-\tau)\left(1-\frac{x(t-\tau)+y(t-\tau)}{K}\right) e^{-m \tau} \\
& -\delta y(t)-\frac{p b}{c} z(t) \\
\leq & r K\left(1-\frac{x(t-\tau)}{K}\right) e^{-m \tau}-\delta y(t)-\frac{p b}{c} z(t) \\
\leq & r K e^{-m \tau}-\rho T(t),
\end{aligned}
$$

where $\rho=\min \{r, \delta, b\}$. Consequently,

$$
T(t) \leq \gamma,
$$

where $\gamma=\max \left\{T(0), r K e^{-m \tau} / \rho\right\}$. This implies that $T(t)$ is bounded on $(\tau,+\infty)$. According to the continuity of $T(t)$ on $[0, \tau]$, we concluded that $T(t)$ is also bounded on $[0, \tau]$. Therefore, $y(t)$ and $z(t)$ are bounded for all $t \geq 0$.

By the third equation of (1) and the boundedness of $y(t)$, we find

$$
\limsup _{t \rightarrow+\infty} v(t) \leq \frac{r K N \delta e^{-m \tau}}{\mu \rho} .
$$

Then, $v(t)$ is bounded. This completes the proof.

Next, we discuss the existence of equilibria of model (1). Denote

$$
\mathscr{R}_{0}=\frac{N K \beta}{n \beta K+\mu} e^{-m \tau}=\frac{1}{\delta} \times \frac{N \delta}{n \beta K+\mu} \times K \beta \times e^{-m \tau},
$$

where $1 / \delta$ is the average life expectancy of the infected tumor cells, $(N \delta) /(n \beta K+\mu)$ is the viral quantity generated from one infected cell during its survival period, $K$ the number of uninfected tumor cells at the beginning of the infection, and $e^{-m \tau}$ is the probability of surviving from time $t-\tau$ to time $t$. Therefore, $\mathscr{R}_{0}$ is the basic reproduction number of model (1) which biologically describes the average number of the newly infected tumor cells generated from one infected cell at the beginning of the infection.

In absence of the innate immune response, system (1) reduced to the model in [10]. Then, (1) always has two equilibria $E_{0}(0,0,0,0)$ and $E_{1}(K, 0,0,0)$ if $\mathscr{R}_{0} \leq 1$. However, model (1) has another equilibrium $E_{2}\left(x_{2}, y_{2}, v_{2}, 0\right)$ if $\mathscr{R}_{0}>1$, where

$$
\begin{aligned}
& x_{2}=\frac{\mu r+K \delta\left(N-n e^{m \tau}\right)(r \alpha-\beta)+\sqrt{\Delta}}{2 r\left(N-n e^{m \tau}\right)\left(\beta e^{-m \tau}+\alpha \delta\right)}, \\
& y_{2}=\frac{r x_{2}\left(K-x_{2}\right)}{r x_{2}+K \delta e^{m \tau}}, \\
& v_{2}=\frac{\delta\left(N-n e^{m \tau}\right)}{\mu} y_{2},
\end{aligned}
$$

with $\Delta=\left[\begin{array}{ll}K \delta\left(N-n e^{m \tau}\right) & (\beta-r \alpha)-\mu r\end{array}\right]^{2}+4 \mu \delta K r e^{m \tau}(N-$ $\left.n e^{m \tau}\right)\left(\beta e^{-m \tau}+\delta \alpha\right)$.

In presence of the innate immune response, we have

$$
\begin{aligned}
x & =\frac{(c r K \alpha-b r \alpha-K c \beta) v+r(c K-b)}{r c(1+\alpha v)}:=\varphi_{1}(v), \\
y & =\frac{b}{c} \\
z & =\frac{b \delta\left(N-n e^{m \tau}\right)-\mu c v}{q c v+n b p e^{m \tau}}:=\varphi_{2}(v), \\
\frac{\beta x v}{1+\alpha v} & =\frac{b}{c} e^{m \tau}(\delta+p z) .
\end{aligned}
$$

Since $z \geq 0$, we have $v \leq \delta b\left(N-n e^{m \tau}\right) / \mu c$. This indicates that there is no biological equilibrium when $v>\delta b\left(N-n e^{m \tau}\right) / \mu c$. Let $F$ be a function defined on the closed interval $\left[0, \delta b\left(N-n e^{m \tau}\right) / \mu c\right]$ as follows:

$$
F(v)=\frac{\beta \varphi_{1}(v) v}{1+\alpha v}-\frac{b}{c} e^{m \tau}\left(\delta+p \varphi_{2}(v)\right) .
$$

Clearly, $F(0)=-b\left(\delta+p \varphi_{2}(0)\right) e^{m \tau}<0$ and

$$
F^{\prime}(v)=\frac{\beta(c K-b)}{c(1+\alpha v)^{2}}-\frac{b}{c} p e^{m \tau} \varphi_{2}^{\prime}(v) .
$$

Since $\quad \varphi_{2}^{\prime}(v)=-b c\left(\mu n p e^{m \tau}+q \delta\left(N-n e^{m \tau}\right)\right) /(q c v+$ $\left.n b p e^{m \tau}\right)^{2}$, we have $F^{\prime}(v)>0$.

When the innate immune response has not been established, we have $c y_{2}-b \leq 0$. Then, we define the reproduction number for the innate immune response as follows:

$$
\mathscr{R}_{1}^{Z}=\frac{c y_{2}}{b},
$$

where $1 / b$ is the average life expectancy of innate immune cells, $c$ is the rate of immune response activation, and $y_{2}$ is the number of infected tumor cells at the steady state $E_{2}$. Hence, $\mathscr{R}_{1}^{Z}$ describes the average number of innate immune cells activated by the infected tumor cells.

If $\mathscr{R}_{1}^{Z}<1$, then $y_{2}<b / c, v_{2}<\delta b\left(N-n e^{m \tau}\right) / \mu c$, and $F(\delta b$ $\left.\left(N-n e^{m \tau}\right) / \mu c\right)<(\delta b / c)\left(\beta x_{2}\left(N-n e^{m \tau}\right) / \mu\left(1+\alpha v_{2}\right)-\right.$ $\left.e^{m \tau}\right)=0$. Then, there is no equilibrium when $\mathscr{R}_{1}^{Z}<1$. 
If $\mathscr{R}_{1}^{Z}>1$, then $y_{2}>b / c, v_{2}>\delta b\left(N-n e^{m \tau}\right) / \mu c$, and $F$ $\left(\delta b\left(N-n e^{m \tau}\right) / \mu c\right)>\delta b / c\left(\beta x_{2}\left(N-n e^{m \tau}\right) / \mu\left(1+\alpha v_{2}\right)-e^{m \tau}\right)$ $=0$. Therefore, model (1) has a unique equilibrium with immune response $E_{3}\left(x_{3}, y_{3}, v_{3}, z_{3}\right)$, where $v_{3} \in(0, \delta b(N-$ $\left.\left.n e^{m \tau}\right) / \mu c\right), \quad x_{3}=(c r K \alpha-b r \alpha-K c \beta) v_{3}+r(c K-b) / r c(1+$ $\left.\alpha v_{3}\right), y_{3}=b / c$, and $z_{3}=b \delta\left(N-n e^{m \tau}\right)-\mu c v_{3} / q c v_{3}+n b p e^{m \tau}$.

By rearranging the above discussions, we have the following theorem.

\section{Theorem 2.}

(i) If $\mathscr{R}_{0} \leq 1$, then model (1) has uniquely two equilibria that are the desired outcome therapy equilibrium $E_{0}(0,0,0,0)$ and the complete failure therapy equilibrium $E_{1}(K, 0,0,0)$

(ii) If $\mathscr{R}_{0}>1$, then model (1) has a unique partial success therapy equilibrium without immune response
$E_{2}\left(x_{2}, y_{2}, v_{2}, 0\right)$ besides $E_{0}$ and $E_{1}$, where $x_{2}=\mu r+$ $K \delta\left(N-n e^{m \tau}\right)(r \alpha-\beta)+\sqrt{\Delta} / 2 r\left(N-n e^{m \tau}\right)\left(\beta e^{-m \tau}\right.$ $+\alpha \delta), \quad y_{2}=r x_{2}\left(K-x_{2}\right) / r x_{2}+K \delta e^{m \tau}, \quad$ and $v_{2}=$ $\delta\left(N-n e^{m \tau}\right) / \mu y_{2}$

(iii) If $\mathscr{R}_{1}^{Z}>1$, then model (1) has a unique partial success therapy equilibrium with immune response $E_{3}\left(x_{3}\right.$, $\left.y_{3}, v_{3}, z_{3}\right)$ besides $E_{0}, E_{1}$, and $E_{2}$, where $v_{3} \in(0, \delta b$ $\left.\left(N-n e^{m \tau}\right) / \mu c\right), \quad x_{3}=(c r K \alpha-b r \alpha-K c \beta) v_{3}+r$ $(c K-b) / r c\left(1+\alpha v_{3}\right), \quad y_{3}=b / c$, and $z_{3}=b \delta(N-$ $\left.n e^{m \tau}\right)-\mu c v_{3} / q c v_{3}+n b p e^{m \tau}$.

\section{Model Analysis and Stability}

To understand the dynamics of the proposed model, we first analyze the local asymptotic stability of equilibria. Let $E(x, y, v, z)$ be an arbitrary equilibrium of model (1). Hence, the characteristic equation at $E$ is given by

$$
\mid \begin{array}{cc}
r\left(1-\frac{2 x+y}{K}\right)-\frac{\beta v}{1+\alpha v}-\lambda & -\frac{r x}{K} \\
\frac{\beta v}{1+\alpha v} e^{-(m+\lambda) \tau} & -\delta-p z-\lambda \\
-\frac{n \beta v}{1+\alpha v} & N \delta \\
0 & c z
\end{array}
$$$$
-\frac{\beta x}{(1+\alpha v)^{2}}
$$$$
0
$$$$
\frac{\beta x}{(1+\alpha v)^{2}} e^{-(m+\lambda) \tau}
$$$$
=0 \text {. }
$$$$
\begin{array}{cc}
-\frac{n \beta x}{(1+\alpha v)^{2}}-\mu-q z-\lambda & -q v \\
0 & c y-b-\lambda
\end{array} \mid=0 .
$$

Afterward, we set $i \psi(\psi>0)$ to be a purely imaginary root of (20). Then,

$$
\left\{\begin{array}{c}
-\psi^{2}+\delta(n \beta K+\mu)=\delta(n \beta K+\mu) \mathscr{R}_{0} \cos (\psi \tau), \\
(n \beta K+\mu+\delta) \psi=-\delta(n \beta K+\mu) \mathscr{R}_{0} \sin (\psi \tau),
\end{array}\right.
$$

which leads to

$$
\psi^{4}+\left[\delta^{2}+(n \beta K+\mu)^{2}\right] \psi^{2}+\delta^{2}(n \beta K+\mu)^{2}\left(1-\mathscr{R}_{0}^{2}\right)=0 .
$$

Denote $\mathcal{S}=\psi^{2}$. Then, the previous equation becomes

$$
\mathcal{S}^{2}+\left[\delta^{2}+(n \beta K+\mu)^{2}\right] \mathcal{S}+\delta^{2}(n \beta K+\mu)^{2}\left(1-\mathscr{R}_{0}^{2}\right)=0,
$$

$$
\begin{aligned}
& (r+\lambda)(b+\lambda)\left[\lambda^{2}+(n \beta K+\mu+\delta) \lambda\right. \\
& \left.+\delta(n \beta K+\mu)\left(1-\mathscr{R}_{0} e^{-\lambda \tau}\right)\right]=0 .
\end{aligned}
$$

Obviously, $\lambda_{1}=-r$ and $\lambda_{2}=-b$ are two negative roots of equation (19). Then, we consider the following transcendental equation:

$$
\lambda^{2}+(n \beta K+\mu+\delta) \lambda+\delta(n \beta K+\mu)\left(1-\mathscr{R}_{0} e^{-\lambda \tau}\right)=0 .
$$

For $\tau=0$ and $\mathscr{R}_{0}<1$, we have $\delta(n \beta K+\mu)\left(1-\mathscr{R}_{0}\right)>0$. Thus, the entire roots of (20) have negative real parts. which has no positive root when $\mathscr{R}_{0}<1$. This implies that $E_{1}$ is locally asymptotically stable if $\mathscr{R}_{0}<1$. In fact, (23) having no positive roots implies that equation (20) does not exhibit any stability switch [12]. This means that the stability of $E_{1}$ for $\tau=0$ is the same as that for $\tau \geq 0$, implying that $E_{1}$ is asymptotically stable for all $\tau \geq 0$

For $\mathscr{R}_{0}>1$, we consider the following function:

$$
f(\lambda)=\lambda^{2}+(n \beta K+\mu+\delta) \lambda+\delta(n \beta K+\mu)\left(1-\mathscr{R}_{0} e^{-\lambda \tau}\right) .
$$

We have $f(0)=\delta(n \beta K+\mu)\left(1-\mathscr{R}_{0}\right)<0$ and $\lim _{\lambda \longrightarrow+\infty}$ $f(\lambda)=+\infty$. Then, the equation $f(\lambda)=0$ has at least one 
positive root when $\mathscr{R}_{0}>1$. This implies that the characteristic equation (19) has at least one positive eigenvalue when $\mathscr{R}_{0}>1$. Therefore, the complete failure therapy equilibrium $E_{1}$ becomes unstable as long as $\mathscr{R}_{0}>1$.

The following result investigates the global stability of the complete failure therapy equilibrium $E_{1}$ when $\mathscr{R}_{0} \leq 1$.

Theorem 5. If $\mathscr{R}_{0} \leq 1$, then the complete failure therapy equilibrium $E_{1}$ is globally asymptotically stable for all $\tau \geq 0$.

Proof. We consider the following functional:

$$
V(t)=e^{m \tau} y(t)+\frac{e^{m \tau}}{N} v(t)+\int_{t-\tau}^{t} \frac{\beta x(s) v(s)}{1+\alpha v(s)} \mathrm{d} s+\frac{p e^{m \tau}}{c} z .
$$

Taking the derivative of $V$ along $t$ of the solutions of (1) delivers

$$
\left.\frac{\mathrm{d} V}{\mathrm{~d} t}\right|_{(1)}=\left(1-\frac{n e^{m \tau}}{N}\right) \frac{\beta x v}{1+\alpha v}-\frac{\mu e^{m \tau}}{N} v-\left(\frac{q}{N}+\frac{p b}{c}\right) z e^{m \tau} .
$$

Seeing that $\limsup _{t \rightarrow \infty} x(t) \leq K$, we deduce that each $\omega$-limit point satisfies $x(t) \leq K$. Hence, it is sufficient to take solutions for which $x(t) \leq K$. Thus,

$$
\left.\frac{\mathrm{d} V}{\mathrm{~d} t}\right|_{(1)} \leq \frac{e^{m \tau}}{N}(n \beta K+\mu)\left(\mathscr{R}_{0}-1\right) v-\left(\frac{q}{N}+\frac{p b}{c}\right) z e^{m \tau} .
$$

Then, $\mathrm{d} V /\left.\mathrm{d} t\right|_{(1)} \leq 0$ when $\mathscr{R}_{0} \leq 1$. Moreover, it is easy to prove that the largest invariant subset of $\{(x, y, v, z) \mid \mathrm{d} V /$ $\mathrm{d} t=0\}$ is the singleton $\left\{E_{1}\right\}$. From LaSalle's invariance principle [13], we conclude that $E_{1}$ is globally asymptotically stable as long as $\mathscr{R}_{0} \leq 1$.

Next, we study the stability of $E_{2}$. In this case, (4) becomes

$$
\left(c y_{2}-b-\lambda\right)\left[\lambda^{3}+a_{1} \lambda^{2}+a_{2} \lambda+a_{3}+\left(b_{1} \lambda+b_{2}\right) e^{-\lambda \tau}\right]=0,
$$

where

$$
\begin{aligned}
& a_{1}=\mu+\delta+\frac{r x_{2}}{K}+\frac{n \beta x_{2}}{\left(1+\alpha v_{2}\right)^{2}}, \\
& a_{2}=\delta \mu+(\mu+\delta) \frac{r x_{2}}{K}+\frac{n \delta \beta x_{2}}{\left(1+\alpha v_{2}\right)^{2}}+\frac{r n \beta x_{2}^{2}}{K\left(1+\alpha v_{2}\right)^{2}}-\frac{n \beta^{2} x_{2} v_{2}}{\left(1+\alpha v_{2}\right)^{3}}, \\
& a_{3}=\frac{r \mu \delta x_{2}}{K}+\frac{r n \beta \delta x_{2}^{2}}{K\left(1+\alpha v_{2}\right)^{2}}-\frac{n \beta^{2} \delta x_{2} v_{2}}{\left(1+\alpha v_{2}\right)^{3}} \\
& b_{1}=\frac{\beta x_{2}}{1+\alpha v_{2}}\left(\frac{r v_{2}}{K}-\frac{N \delta}{1+\alpha v_{2}}\right) e^{-m \tau}, \\
& b_{2}=\frac{\beta x_{2}}{1+\alpha v_{2}}\left(\frac{r \mu v_{2}}{K}+\frac{N \beta \delta v_{2}}{\left(1+\alpha v_{2}\right)^{2}}-\frac{N \delta r x_{2}}{K\left(1+\alpha v_{2}\right)}\right) e^{-m \tau} .
\end{aligned}
$$

Clearly, $\lambda_{1}=c y_{2}-b$ is a root of (28). If $\mathscr{R}_{1}^{Z}>1$, then $\lambda_{1}>0$ and $E_{2}$ is unstable. However, $\lambda_{1}<0$ if $\mathscr{R}_{1}^{Z}<1$. In this case, we study the roots of the following equation:

$$
\lambda^{3}+a_{1} \lambda^{2}+a_{2} \lambda+a_{3}+\left(b_{1} \lambda+b_{2}\right) e^{-\lambda \tau}=0 .
$$

The general form of this transcendental characteristic equation was investigated by Beretta and Kuang in [14].

For $\tau=0$, (30) becomes

$$
\lambda^{3}+a_{1} \lambda^{2}+\left(a_{2}+b_{1}\right) \lambda+a_{3}+b_{2}=0 .
$$

Since $a_{1}>0$ and $a_{3}+b_{2}=(N-n) \beta^{2} \delta x_{2} v_{2} /\left(1+\alpha v_{2}\right)^{3}+$ $r \mu(\alpha \delta+\beta) x_{2} v_{2} / K\left(1+\alpha v_{2}\right)>0$, we deduce by applying the Routh-Hurwitz criterion that $E_{2}$ is locally asymptotically stable if $a_{1}\left(a_{2}+b_{1}\right)-\left(a_{3}+b_{2}\right)>0$.

Let $i \vartheta(\vartheta>0)$ be a root of $(30)$. Then,

$$
\left\{\begin{array}{l}
a_{1} \vartheta^{2}-a_{3}=b_{1} \vartheta \sin (\vartheta \tau)+b_{2} \cos (\vartheta \tau), \\
-\vartheta^{3}+a_{2} \vartheta=-b_{1} \vartheta \cos (\vartheta \tau)+b_{2} \sin (\vartheta \tau) .
\end{array}\right.
$$

Hence,

$$
\vartheta^{6}+\left(a_{1}^{2}-2 a_{2}\right) \vartheta^{4}+\left(a_{2}^{2}-2 a_{1} a_{3}-b_{1}^{2}\right) \vartheta^{2}+a_{3}^{2}-b_{2}^{2}=0,
$$

which reduces to

$$
g(\mathcal{S}):=\mathcal{S}^{3}+q_{2} \mathcal{S}^{2}+q_{1} \mathcal{S}+q_{0}=0,
$$

where $\delta=\vartheta^{2}, \quad q_{2}=a_{1}^{2}-2 a_{2}, \quad q_{1}=a_{2}^{2}-2 a_{1} a_{3}-b_{1}^{2}$, and $q_{0}=a_{3}^{2}-b_{2}^{2}$. By an analogical discussion as in [10], let $\Delta=$ $q_{2}^{2}-3 q_{1}$ and $\mathcal{\delta}^{*}=\sqrt{\Delta}-q_{2} / 3$. Hence, we consider the following assertions:

$$
\begin{aligned}
& \left(i_{1}\right) q_{0} \geq 0 \text { and } \Delta \leq 0 \\
& \left(i_{2}\right) q_{0} \geq 0, \Delta>0 \text {, and } \mathcal{S}^{*} \leq 0 \\
& \left(i_{3}\right) q_{0} \geq 0, \Delta>0 \text {, and } g\left(\mathcal{S}^{*}\right)>0
\end{aligned}
$$

Therefore, we have the following result.

Theorem 6. Assume $\mathscr{R}_{0}>1$.

(1) If $\mathscr{R}_{1}^{Z}<1, a_{1}\left(a_{2}+b_{1}\right)-\left(a_{3}+b_{2}\right)>0$, and one of the conditions $\left(i_{1}\right)-\left(i_{3}\right)$ holds, then the partial success therapy equilibrium without immune response $E_{2}$ is locally asymptotically stable for any time delay $\tau \geq 0$

(2) If $\mathscr{R}_{1}^{Z}>1$, then $E_{2}$ is unstable.

Assume that equation (34) has positive roots. Without loss of generality, we assume that (34) has three positive solutions named $\delta_{1}, \delta_{2}$, and $\delta_{3}$ which are ordered as follows: $\mathcal{S}_{1}<\mathcal{S}_{2}<\mathcal{S}_{3}$. It follows that equation (33) admits three positive solutions that are

$$
\begin{aligned}
& \vartheta_{1}=\sqrt{\mathcal{S}_{1}}, \\
& \vartheta_{2}=\sqrt{\mathcal{S}_{2}} \text { and } \vartheta_{3}=\sqrt{\mathcal{S}_{3}} .
\end{aligned}
$$


By (32), we get

$$
\tau_{k}^{j}=\frac{1}{\vartheta_{j}} \arccos \left(\frac{b_{2}\left(a_{1} \vartheta_{j}^{2}-a_{3}\right)+b_{1} \vartheta_{j}^{2}\left(\vartheta_{j}^{2}-a_{2}\right)}{b_{2}^{2}+b_{1}^{2} \vartheta_{j}^{2}}\right)+\frac{2 k \pi}{\vartheta_{j}},
$$

where $j=1,2,3$ and $k \in \mathrm{IN}$. Therefore, $\pm i \vartheta_{j}$ is a pair of purely imaginary roots of (30) with $\tau=\tau_{k}^{j}$. Let

$$
\tau_{0}=\tau_{0}^{j_{0}}=\min _{j \in\{1,2,3\}}\left\{\tau_{0}^{j}\right\}, \vartheta_{0}=\vartheta_{j_{0}} .
$$

We $\operatorname{set} \lambda(\tau)=\varsigma(\tau)+i \vartheta(\tau)$ to be the root of equation (30) satisfying $\varsigma\left(\tau_{k}^{j}\right)=0$ and $\vartheta\left(\tau_{k}^{j}\right)=\vartheta_{j}$. Differentiating (30) with respect to $\tau$, we get

$$
\left(\frac{\mathrm{d} \lambda}{\mathrm{d} \tau}\right)^{-1}=\frac{3 \lambda^{2}+2 a_{1} \lambda+a_{2}+b_{1} e^{-\lambda \tau}}{\lambda\left(b_{1} \lambda+b_{2}\right) e^{-\lambda \tau}}-\frac{\tau}{\lambda}
$$

Thus,

$$
\begin{aligned}
\left.\operatorname{Re}\left(\frac{\mathrm{d} \lambda}{\mathrm{d} \tau}\right)^{-1}\right|_{\tau=\tau_{k}^{j}} & =\frac{3 \vartheta_{j}^{4}+2\left(a_{1}^{2}-2 a_{2}\right) \vartheta_{j}^{2}+a_{2}^{2}-2 a_{1} a_{3}-b_{1}^{2}}{b_{1}^{2} \vartheta_{j}^{2}+b_{2}^{2}} \\
& =\frac{g^{\prime}\left(\vartheta_{j}^{2}\right)}{b_{1}^{2} \vartheta_{j}^{2}+b_{2}^{2}} .
\end{aligned}
$$

It is not difficult to find out that $g^{\prime}\left(\vartheta_{j}^{2}\right) \neq 0$ for all $j=1,2,3$. Then, the transversality condition holds and we get the following result.

Theorem 7. Assume $\mathscr{R}_{1}^{Z}<1<\mathscr{R}_{0}$ and $a_{1}\left(a_{2}+b_{1}\right)-\left(a_{3}+\right.$ $\left.b_{2}\right)>0$ hold.

If either $q_{0}<0$ or $q_{0} \geq 0, \Delta>0, \delta^{*}>0$, and $g\left(\delta^{*}\right) \leq 0$, then $E_{2}$ is locally asymptotically stable for all $\tau \in\left[0, \tau_{0}\right)$ and becomes unstable when $\tau>\tau_{0}$. Moreover, model (1) undergoes a Hopf bifurcation at $E_{2}$ when $\tau=\tau_{k}^{j}$, for $j=1,2,3$ and $k \in I N$.

Remark 1. Theorem 7 shows that the delay $\tau$ can cause the partial success therapy equilibrium without immune response $E_{2}$ to gain or lose its stability. In addition, periodic solutions appear when the value of this delay is equal to a critical value.

Finally, we discuss the stability of the partial success therapy equilibrium with immune response $E_{3}$ when $\mathscr{R}_{1}^{Z}>1$. In this case, (17) becomes

$$
\lambda^{4}+c_{3} \lambda^{3}+c_{2} \lambda^{2}+c_{1} \lambda+c_{0}+\left(d_{2} \lambda^{2}+d_{1} \lambda+d_{0}\right) e^{-\lambda \tau}=0,
$$

where

$$
\begin{aligned}
& c_{3}=\mu+\delta+(p+q) z_{3}+\frac{r x_{3}}{K}+\frac{n \beta x_{3}}{\left(1+\alpha v_{3}\right)^{2}}, \\
& c_{2}=b p z_{3}+\left(\mu+\delta+(p+q) z_{3}+\frac{n \beta x_{3}}{\left(1+\alpha v_{3}\right)^{2}}\right) \frac{r x_{3}}{K}+\left(\delta+p z_{3}\right)\left(\mu+q z_{3}+\frac{n \beta x_{3}}{\left(1+\alpha v_{3}\right)^{2}}\right)-\frac{n \beta^{2} x_{3} v_{3}}{\left(1+\alpha v_{3}\right)^{3}}, \\
& c_{1}=b p z_{3}\left(\left(\mu+q z_{3}\right)+\frac{n \beta x_{3}}{\left(1+\alpha v_{3}\right)^{2}}\right)+\frac{r x_{3}}{K}\left[b p z_{3}+\left(\delta+p z_{3}\right)\left(\mu+q z_{3}+\frac{n \beta x_{3}}{\left(1+\alpha v_{3}\right)^{2}}\right)\right]-\frac{n \beta^{2} x_{3} v_{3}}{\left(1+\alpha v_{3}\right)^{3}}\left(\delta+p z_{3}\right), \\
& c_{0}=b p z_{3}\left[\frac{r x_{3}}{K}\left(\mu+q z_{3}+\frac{n \beta x_{3}}{\left(1+\alpha v_{3}\right)^{2}}\right)-\frac{n \beta^{2} x_{3} v_{3}}{\left(1+\alpha v_{3}\right)^{3}}\right] \\
& d_{2}=\frac{\beta x_{3}}{1+\alpha v_{3}}\left(\frac{r v_{3}}{K}-\frac{N \delta}{1+\alpha v_{3}}\right) e^{-m \tau}, \\
& d_{1}=\left[\frac{\beta N \delta x_{3}}{\left(1+\alpha v_{3}\right)^{2}}\left(\frac{\beta v_{3}}{1+\alpha v_{3}}-\frac{r x_{3}}{K}\right)+\frac{\beta x_{3} v_{3}}{1+\alpha v_{3}}\left(\frac{r}{K}\left(\mu+q z_{3}\right)+\frac{q c z_{3}}{1+\alpha v_{3}}\right)\right] e^{-m \tau}, \\
& d_{0}=\frac{\beta q c x_{3} v_{3} z_{3}}{\left(1+\alpha v_{3}\right)^{2}}\left(\frac{r x_{3}}{K}-\frac{\beta v_{3}}{1+\alpha v_{3}}\right) e^{-m \tau} .
\end{aligned}
$$


The above equation is the same as that analyzed by Hattaf in [9]. Then, let $i \phi(\phi>0)$ be a root of (41). We have

$$
\left\{\begin{array}{l}
\phi^{4}-c_{2} \phi^{2}+c_{0}=\left(d_{2} \phi^{2}-d_{0}\right) \cos (\phi \tau)-b_{1} \phi \sin (\phi \tau), \\
-c_{3} \phi^{3}+c_{1} \phi=-d_{1} \phi \cos (\phi \tau)-\left(d_{2} \phi^{2}-d_{0}\right) \sin (\phi \tau),
\end{array}\right.
$$

which can be reduced to

$$
\begin{aligned}
\phi^{8} & +\left(c_{3}^{2}-2 c_{2}\right) \phi^{6}+\left(c_{2}^{2}-d_{2}^{2}+2 c_{0}-2 c_{1} c_{3}\right) \phi^{4} \\
& +\left(c_{1}^{2}-d_{1}^{2}-2 c_{0} c_{2}+2 d_{0} d_{2}\right) \phi^{2}+c_{0}^{2}-d_{0}^{2}=0 .
\end{aligned}
$$

Let $u=\phi^{2}$. Then, (43) becomes

$$
h(u):=u^{4}+p_{3} u^{3}+p_{2} u^{2}+p_{1} u+p_{0}=0,
$$

where $p_{3}=c_{3}^{2}-2 c_{2}, \quad p_{2}=c_{2}^{2}-d_{2}^{2}+2 c_{0}-2 c_{1} c_{3}, \quad p_{1}=c_{1}^{2}-$ $d_{1}^{2}-2 c_{0} c_{2}+2 d_{0} d_{2}$, and $p_{0}=c_{0}^{2}-d_{0}^{2}$. Clearly, if $p_{0}<0$, equation (44) admits at least one positive root. Additionally, we have

$$
h^{\prime}(u)=4 u^{3}+3 p_{3} u^{2}+2 p_{2} u+p_{1}=0 .
$$

According to Cardano's formula, the cubic roots of (45) can be written as follows:

$$
\begin{aligned}
& u_{1}=\sqrt[3]{-\frac{\mathbb{Q}}{2}+\sqrt{\bar{\Delta}}}+\sqrt[3]{-\frac{\mathbb{Q}}{2}+\sqrt{\bar{\Delta}}}-\frac{p_{3}}{4}, \\
& u_{2}=j \sqrt[3]{-\frac{\mathbb{Q}}{2}+\sqrt{\bar{\Delta}}}+j^{2} \sqrt[3]{-\frac{\mathbb{Q}}{2}-\sqrt{\bar{\Delta}}}-\frac{p_{3}}{4}, \\
& u_{3}=j^{2} \sqrt[3]{-\frac{\mathbb{Q}}{2}+\sqrt{\bar{\Delta}}}+j \sqrt[3]{-\frac{\mathbb{Q}}{2}-\sqrt{\bar{\Delta}}}-\frac{p_{3}}{4},
\end{aligned}
$$

where

$$
\begin{aligned}
\mathscr{P} & =\frac{8 p_{2}-3 p_{3}^{2}}{16}, \\
\mathscr{Q} & =\frac{p_{3}^{3}-4 p_{3} p_{2}+8 p_{1}}{32}, \\
\bar{\Delta} & =\left(\frac{\mathscr{P}}{3}\right)^{3}+\left(\frac{\mathscr{Q}}{2}\right)^{2}, \\
j & =-\frac{1}{2}+i \frac{\sqrt{3}}{2} .
\end{aligned}
$$
(44).

Hence, we discuss the existence of real positive roots of

(i) When $\bar{\Delta}>0$, (44) has only a real root $u_{1}$ and the other two roots are conjugate complex numbers. Thus,

$$
h^{\prime}(u)=4\left(u-u_{1}\right)\left(u^{2}-2 \operatorname{Re}\left(u_{2}\right) z+\left|u_{2}\right|^{2}\right) .
$$

It follows that $h$ admits a unique strict global minimum at $u=u_{1}$, and it is because $u^{2}-2 \operatorname{Re}\left(u_{2}\right) z+\left|u_{2}\right|^{2}>0$ for all $u \in$ IR.

(ii) When $\bar{\Delta}=0$, all roots are real with $u_{1}=3 \mathscr{Q} / \mathscr{P}$ $p_{3} / 4$ and $u_{2}=u_{3}=3 \mathscr{Q} / 2 \mathscr{P}-p_{3} / 4$. Hence,

$$
h^{\prime}(u)=4\left(u-u_{1}\right)\left(u-u_{2}\right)^{2} \text {. }
$$

Thus, $h$ reaches its strict global minimum at $u=u_{1}$. We conclude that if $p_{0} \geq 0$ and $\bar{\Delta} \geq 0$, then equation (44) has a positive root if and only if $u_{1}>0$ and $h\left(u_{1}\right) \leq 0$.

(iii) When $\bar{\Delta}<0$, the entire roots are real and distinct. In this case, we have

$$
h^{\prime}(u)=4\left(u-u_{1}\right)\left(u-u_{2}\right)\left(u-u_{3}\right) .
$$

By analogical reasoning, we deduce that if $p_{0} \geq 0$ and $\bar{\Delta}<0$, then equation (44) has positive root if and only if there exists at least one $u^{*} \in\left\{u_{1}, u_{2}, u_{3}\right\}$ verifying $u^{*}>0$ and $h\left(u^{*}\right) \leq 0$.

A summary of the above analysis leads to the following lemma.

\section{Lemma 1}

(i) If $p_{0}<0$, then equation (44) has at least one positive root

(ii) If $p_{0} \geq 0$ and $\bar{\Delta}<0$, then equation (44) has a positive root if and only if $u_{1} \geq 0$ and $h\left(u_{1}\right) \leq 0$

(iii) If $p_{0} \geq 0$ and $\bar{\Delta}<0$, then equation (44) has a positive root if and only if there exists at least one $u^{*} \in\left\{u_{1}, u_{2}, u_{3}\right\}$ verifying $u^{*}>0$ and $h\left(u^{*}\right) \leq 0$.

Based on Lemma 1, we set the following conditions:

$$
\begin{aligned}
& \left(\mathrm{H}_{1}\right) p_{0}<0 \\
& \left(\mathrm{H}_{2}\right) p_{0} \geq 0, \bar{\Delta} \geq 0, u_{1}>0, \text { and } h\left(u_{1}\right) \leq 0 \\
& \left(\mathrm{H}_{3}\right) p_{0} \geq 0, \bar{\Delta}<0, \text { and there exists at least one } \\
& u^{*} \in\left\{u_{1}, u_{2}, u_{3}\right\} \text { verifying } u^{*}>0 \text { and } h\left(u^{*}\right) \leq 0,
\end{aligned}
$$

If conditions $\left(\mathrm{H}_{1}\right)-\left(\mathrm{H}_{3}\right)$ are not fulfilled, then equation (44) has no positive solutions. Thus, equation (40) has no purely imaginary roots. Consequently, the partial success therapy equilibrium with immune response $E_{3}\left(x_{3}, y_{3}, v_{3}, z_{3}\right)$ is locally asymptotically stable for all $\tau \geq 0$. In this case, the presence of Hopf bifurcation is not achievable.

Next, we assume that one of the conditions $\left(\mathrm{H}_{1}\right)-\left(\mathrm{H}_{3}\right)$ is fulfilled; then equation (44) admits at least one positive solution. Let $d \in\{1,2,3,4\}$ be the number of positive roots of 
(44). Denote these $d$ positive roots by $u_{k}$. Then, equation (43) has $d$ positive solutions $\phi_{k}=\sqrt{u_{k}}, k=1,2, \ldots, d$. Therefore, from (42), we obtain

$$
\begin{aligned}
\tau_{\eta}^{k}= & \frac{1}{\phi_{k}} \arccos \left(\frac{\left(\phi_{k}^{4}-c_{2} \phi_{k}^{2}+c_{0}\right)\left(b_{2} \phi_{k}^{2}-b_{0}\right)+b_{1} \phi_{k}^{2}\left(c_{3} \phi_{k}^{4}-c_{1}\right)}{b_{1} \phi_{k}^{2}+\left(b_{2} \phi_{k}^{2}-b_{0}\right)^{2}}\right) \\
& +\frac{2 \eta \pi}{\phi_{k}}
\end{aligned}
$$

where $k=1,2, \ldots, d$ and $\eta \in \mathrm{IN}$. We deduce that $\pm i \phi_{k}$ is a pair of purely imaginary roots of (40) with $\tau=\tau_{\eta}^{k}$. Define

$$
\tau_{0}=\tau_{0}^{k_{0}}=\min _{k \in\{1,2, \ldots, d\}}\left\{\tau_{0}^{k}\right\}, \phi_{0}=\phi_{k_{0}}
$$

We set $\lambda(\tau)=\varsigma(\tau)+i \phi(\tau)$ to be the root of equation (40) such that $\varsigma\left(\tau_{\eta}^{k}\right)=0$ and $\phi\left(\tau_{\eta}^{k}\right)=\phi_{k}$. Considering $\varsigma$ as a function of $\tau$ and differentiating both sides of (40) with respect to $\tau$ lead to

$$
\left(\frac{\mathrm{d} \lambda}{\mathrm{d} \tau}\right)^{-1}=\frac{4 \lambda^{3}+3 c_{3} \lambda^{2}+2 c_{2} \lambda+c_{1}+\left(2 d_{2} \lambda+d_{1}\right) e^{-\lambda \tau}}{\lambda\left(d_{2} \lambda^{2}+d_{1} \lambda+d_{0}\right) e^{-\lambda \tau}}-\frac{\tau}{\lambda}
$$

This implies that

$$
\left.\operatorname{Re}\left(\frac{\mathrm{d} \lambda}{\mathrm{d} \tau}\right)^{-1}\right|_{\tau=\tau_{\eta}^{k}}=\frac{4 \phi_{k}^{6}+3 p_{3} \phi_{k}^{4}+2 p_{2} \phi_{k}^{2}+p_{1}}{b_{1} \phi_{k}^{2}+\left(b_{2} \phi_{k}^{2}-b_{0}\right)^{2}},=\frac{h^{\prime}\left(\phi_{k}^{2}\right)}{b_{1}^{2} \phi_{k}^{2}+b_{2}^{2}}
$$

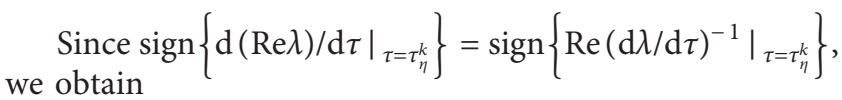

$$
\begin{aligned}
& \operatorname{sign}\left\{\mathrm{d}(\operatorname{Re} \lambda) /\left.\mathrm{d} \tau\right|_{\tau=\tau_{\eta}^{k}}\right\} \\
& \quad=\operatorname{sign}\left\{h^{\prime}\left(u_{k}\right)\right\}=\operatorname{sign}\left\{4 \phi_{k}^{6}+3 p_{3} \phi_{k}^{4}+2 p_{2} \phi_{k}^{2}+p_{1}\right\} .
\end{aligned}
$$

Therefore, based on the above analysis, we claim the following result.

\section{Theorem 8.}

Assume that $\mathscr{R}_{1}^{Z}>1$.

(i) If conditions $\left(H_{1}\right)-\left(H_{3}\right)$ are not fulfilled, then the partial success therapy equilibrium with immune response $E_{3}$ is locally asymptotically stable for all $\tau \geq 0$.

(ii) If one of the conditions $\left(H_{1}\right)-\left(H_{3}\right)$ is fulfilled, then the partial success therapy equilibrium with immune response $E_{3}$ is locally asymptotically stable for any time delay $\tau \in\left[0, \tau_{0}\right)$ and becomes unstable when $\tau>\tau_{0}$. Furthermore, if $h^{\prime}\left(\phi_{0}^{2}\right) \neq 0$, then the transversality condition holds and model (1) undergoes a Hopf bifurcation at $E_{3}$ when $\tau=\tau_{0}$.
Based on Lemma 4.3 in [9], we easily deduce the following theorem.

\section{Theorem 9.}

Assume that $\mathscr{R}_{1}^{Z}>1$.

(i) If equation (44) admits only one positive and simple root $u_{1}$, then $E_{3}$ is locally asymptotically stable for $\tau \in\left[0, \tau_{0}^{1}\right)$ and becomes unstable for $\tau>\tau_{0}^{1}$. Furthermore, a Hopf bifurcation appears when $\tau=\tau_{\eta}^{1}$, $\eta \in I N$.

(ii) If equation (44) admits only two positive and simple roots $u_{1}, u_{2}$ which are ordered as $u_{2}<u_{1}$, then there exist a finite number of intervals such that if the delay $\tau$ is fixed in these intervals, the equilibrium $E_{3}$ is locally asymptotically stable, while unstable if $\tau$ not belonging to ones. In this case, $E_{3}$ switches from stability to instability.

(iii) If equation (44) admits at least three positive and simple roots, then there exists a least one stability switch.

Remark 2. Theorems 8 and 9 show that when the delay $\tau$ is considered, the partial success equilibrium with immune response $E_{3}$ can lose or gain its stability and rich dynamical behaviors occur including Hopf bifurcation and stability switches.

\section{Discussion and Conclusion}

In this paper, we have proposed and analyzed a virological model for cancer therapy with effects of saturation, innate immune response, and delay that biologically represents the time needed for infected tumor cells to produce new virions after viral entry. We first proved the positivity and the boundedness of solutions and discussed the existence of equilibria by means of two threshold parameters that are the basic reproduction number denoted by $\mathscr{R}_{0}$ and the reproduction number for innate immune response labeled by $\mathscr{R}_{1}^{Z}$ which represents the average number of innate immune cells activated by damaged tumor cells. More accurately, the proposed model has uniquely (i) two equilibria, the desired outcome therapy equilibrium $E_{0}$ and the complete failure therapy equilibrium $E_{1}$ if $\mathscr{R}_{0} \leq 1$; (ii) three equilibria: $E_{0}$, $E_{1}$, and the partial success therapy equilibrium without immune response $E_{2}$ if $\mathscr{R}_{1}^{Z} \leq 1<\mathscr{R}_{0}$; and (iii) four equilibria: $E_{0}, E_{1}, E_{2}$, and the partial success therapy equilibrium with immune response $E_{3}$ if $\mathscr{R}_{1}^{Z}>1$. We have demonstrated that $E_{0}$ is always unstable and $E_{1}$ is globally asymptotically stable if $\mathscr{R}_{0} \leq 1$ and becomes unstable if $\mathscr{R}_{0}>1$. Additionally, the stability of $E_{2}$ and $E_{3}$, Hopf bifurcation, and stability switches are analyzed rigorously. Furthermore, our model generalizes those in $[7,10]$ and our analytical results show that the delay in infection with oncolytic viruses can lead to the loss or stability of both equilibria $E_{2}$ and $E_{3}$. 


\section{Data Availability}

The data used to support the findings of this study are available from the corresponding author upon request.

\section{Conflicts of Interest}

The authors declare that they have no conflicts of interest.

\section{References}

[1] E. Kelly and S. J. Russell, "History of oncolytic viruses: genesis to genetic engineering," Molecular Therapy, vol. 15, no. 4, pp. 651-659, 2007.

[2] T. C. Liau, E. Galanis, and D. Kirn, "Clinical trial results with oncolytic virotherapy: a century of promise, a decade of progress," Journal of Nature Clinical Practice Oncology, vol. 4, pp. 101-117, 2007.

[3] S. J. Russel, K. W. Pengl, and J. C. Bell, "Oncolytic virotherapy," Journal of Nature Biotechnology, vol. 30, pp. 658670, 2012.

[4] J. Malinzi, P. Sibanda, and H. Mambili-Mamboundou, "Analysis of virotherapy in solid tumor invasion," Mathematical Biosciences, vol. 263, pp. 102-110, 2015.

[5] A. Nguyen, L. Ho, and Y. Wan, "Chemotherapy and oncolytic virotherapy: advanced tactics in the war against cancer," Frontiers in Oncology, vol. 4, p. 145, 2014.

[6] E. Binz and L. M. Ulrich, "Chemovirotherapy: combining chemotherapeutic treatment with oncolytic virotherapy," Oncolytic Virotherapy, vol. 4, pp. 39-48, 2015.

[7] T. A. Phan and J. P. Tian, "The role of the innate immune system in oncolytic virotherapy," Computational and Mathematical Methods in Medicine, vol. 2017, pp. 1-17, 2017.

[8] K. S. Kim, S. Kim, and I. H. Jung, "Hopf bifurcation analysis and optimal control of treatment in a delayed oncolytic virus dynamics," Mathematics and Computers in Simulation, vol. 149, pp. 1-16, 2018.

[9] K. Hattaf, "Global stability and Hopf bifurcation of a generalized viral infection model with multi-delays and humoral immunity," Physica A: Statistical Mechanics and Its Applications, vol. 545, Article ID 123689, 2020.

[10] A. Nouni, K. Hattaf, and N. Yousfi, "Dynamics of a mathematical model for cancer therapy with oncolytic viruses," Communications in Mathematical Biology and Neuroscience, vol. 1, no. 6, pp. 1-12, 2019.

[11] J. Hale and S. M. Verduyn Lunel, Introduction to Functional Differential Equations, Springer-Verlag, New York, NY, USA, 1993.

[12] H. I. Freedman and Y. Kuang, "Stability switches in linear scalar neutral delay equations," Funkcialaj Ekvacioj, vol. 34, pp. 187-209, 1991.

[13] J. P. LaSalle, "The stability of dynamical systems," in Proceedings of the Regional Conference Series in Applied Mathematics, SIAM, Philadelphia, PA, USA, July 1976.

[14] E. Beretta and Y. Kuang, "Geometric stability switch criteria in delay differential systems with delay dependent parameters," SIAM Journal on Mathematical Analysis, vol. 33, no. 5, pp. 1144-1165, 2002. 